\title{
Isolation of potent SARS-CoV-2 neutralizing antibodies and protection from disease in a small animal model
}

\author{
Thomas F. Rogers, ${ }^{1,2 *}$, Fangzhu Zhao ${ }^{1,3,4 *}$, Deli Huang1*, Nathan Beutler ${ }^{1 *}$, Alison Burns ${ }^{1,3,4}$, Wan-ting He ${ }^{1,3,4}$, \\ Oliver Limbo ${ }^{3,5}$, Chloe Smith ${ }^{1,3}$, Ge Song ${ }^{1,3,4}$, Jordan Woeh ${ }^{3,5}$, Linlin Yang', Robert K. Abbott ${ }^{4,6}$, Sean \\ Callaghan $^{1,3,4}$, Elijah Garcia ${ }^{1}$, Jonathan Hurtado ${ }^{1,4,7}$, Mara Parren', Linghang Peng1, Sydney Ramirez ${ }^{6}$, James \\ Ricketts $^{1}$, Michael J. Ricciardi ${ }^{8}$, Stephen A. Rawlings ${ }^{2}$, Nicholas C. Wu ${ }^{9}$, Meng Yuan ${ }^{9}$, Davey M. Smith ${ }^{2}$, David \\ Nemazee $^{1}$, John R. Teijaro ${ }^{1}$, James E. Voss ${ }^{1}$, Ian A. Wilson ${ }^{3,4,9}$, Raiees Andrabi ${ }^{1,3,4}$, Bryan Briney ${ }^{1,4,7}$, Elise \\ Landais $^{1,3,4,5}$, Devin Sok ${ }^{1,3,4,5}+$, Joseph G. Jardine ${ }^{3,5}+$, Dennis R. Burton ${ }^{1,3,4,10} \dagger$
}

${ }^{1}$ Department of Immunology and Microbiology, The Scripps Research Institute, La Jolla, CA 92037, USA. ²Division of Infectious Diseases, Department of Medicine, University of California, San Diego, La Jolla, CA 92037, USA. ${ }^{3}$ AVI Neutralizing Antibody Center, The Scripps Research Institute, La Jolla, CA 92037, USA ${ }^{4}$ Consortium for HIV/AIDS Vaccine Development (CHAVD), The Scripps Research Institute, La Jolla, CA 92037, USA. International AIDS Vaccine Initiative (IAVI), New York, NY10004, USA. ${ }^{6}$ Center for Infectious Disease and Vaccine Research, La Jolla Institute for Immunology (LJI), La Jolla, CA 92037, USA. ${ }^{7}$ Center for Viral Systems Biology, The Scripps Research Institute, La Jolla, CA 92037, USA. ${ }^{8}$ George Washington University, Washington, DC 20052, USA. ${ }^{9}$ Department of Integrative Structural and Computational Biology, The Scripps Research Institute, La Jolla, CA 92037, USA. ${ }^{10}$ Ragon Institute of Massachusetts General Hospital, Massachusetts Institute of Technology, and Harvard University, Cambridge, MA 02139, USA.

*These authors contributed equally to this work.

†Corresponding author. Email: dsok@iavi.org (D.S.); jjardine@iavi.org (J.G.J.); burton@scripps.edu (D.R.B.)

Countermeasures to prevent and treat COVID-19 are a global health priority. We enrolled a cohort of SARSCoV-2-recovered participants, developed neutralization assays to interrogate antibody responses, adapted our high-throughput antibody generation pipeline to rapidly screen over 1800 antibodies, and established an animal model to test protection. We isolated potent neutralizing antibodies (nAbs) to two epitopes on the receptor binding domain (RBD) and to distinct non-RBD epitopes on the spike (S) protein. We showed that passive transfer of a nAb provides protection against disease in high-dose SARS-CoV-2 challenge in Syrian hamsters, as revealed by maintained weight and low lung viral titers in treated animals. The study suggests a role for nAbs in prophylaxis, and potentially therapy, of COVID-19. The nAbs define protective epitopes to guide vaccine design.

The novel coronavirus disease (COVID-19) has had devastating global health consequences and there is currently no cure and no licensed vaccine. Neutralizing antibodies (nAbs) to the causative agent of the disease, severe acute respiratory syndrome coronavirus-2 (SARS-CoV-2), represent potential prophylactic and therapeutic options and could help guide vaccine design. Indeed, a $\mathrm{nAb}$ to another respiratory virus, respiratory syncytial virus (RSV), is in widespread clinical use prophylactically to protect vulnerable infants (1). Furthermore, nAbs prevent death from the emerging Ebola virus in macaques, even when given relatively late in infection, and thus have been proposed for use in humans in outbreaks (2, 3). Generally, nAbs with outstanding potency ("super-antibodies") (4) can be isolated by deeply mining antibody responses of a sampling of infected donors. Outstanding potency together with engineering to extend antibody halflife from weeks to many months brings down the effective costs of Abs and suggests more opportunities for prophylactic intervention. At the same time, outstanding potency can permit anti-viral therapeutic efficacy that is not observed for less potent antibodies (4). Here, we present the isolation of highly potent nAbs to SARS-CoV-2 and demonstrate their in vivo protective efficacy in a small animal model, suggesting their potential utility as a medical countermeasure.

To interrogate the antibody response against SARS-CoV-2 and discover nAbs, we adapted our pipeline to rapidly isolate and characterize monoclonal antibodies (mAbs) from convalescent donors (Fig. 1). Briefly, a cohort of previously swabpositive SARS-CoV-2 donors was recruited for peripheral blood mononuclear cell (PBMC) and plasma collection. In parallel, we developed both live replicating and pseudovirus neutralization assays using a HeLa-ACE2 (Angiotensin-Converting Enzyme-2) cell line that gave robust and reproducible virus titers. Convalescent serum responses were evaluated for neutralization activity against SARS-CoV-1 and SARS-CoV-2, and eight donors were selected for mAb discovery. Single antigen-specific memory B cells were sorted, and their corresponding variable genes were recovered and cloned using a high-throughput production system that enabled antibody expression and characterization in under two weeks. Promising mAbs were advanced for further biophysical characterization and in vivo testing. 


\section{Development of viral neutralization assays}

Two platforms were established to evaluate plasma neutralization activity against SARS-CoV-2, one using replicationcompetent virus and another using pseudovirus (PSV). VeroE6 cells were first used as target cells for neutralization assays, but this system was relatively insensitive at detecting replicating virus compared to a HeLa cell line that stably expressed the cell surface ACE2 receptor (fig. S1A). The HeLaACE2 target cells gave reproducible titers and were used for the remainder of the study. In certain critical instances, HeLa-ACE2 and Vero cells were compared.

The live replicating virus assay used the Washington strain of SARS-CoV-2, USA-WA1/2020 (BEI Resources NR52281) and was optimized to a 384-well format to measure plaque formation. In parallel, a PSV assay was established for both SARS-CoV-1 and SARS-CoV-2 using murine leukemia virus (MLV)-based PSV (5). The assay used single cycle infectious viral particles bearing a firefly luciferase reporter for high-throughput screening. Unlike MLV-PSV, which buds at the plasma membrane, coronaviruses assemble in the ERGolgi intermediate compartment, so the $\mathrm{C}$ terminus of the SARS-CoV-1 Spike protein (S protein) contains an ER retrieval signal (6). The alignment of SARS-CoV-1 and SARSCoV-2 S proteins showed that this ER retrieval signal is conserved in SARS-CoV-2 (fig. S1B). To prepare high titers of infectious MLV-CoV-1 and SARS-CoV-2 PSV particles, various truncations of SARS-CoV-1 and SARS-CoV-2 S protein were expressed in which the ER retrieval signal was removed to improve exocytosis of the virus. Pseudovirion versions carrying SARS-CoV1-S $\Delta 28$ and SARS-CoV2-S $\Delta 18 \mathrm{~S}$ protein efficiently transduced ACE2-expressing target cells, but not control HeLa or A549 cells (fig. S1C). Control VSV-G pseudotyped virions showed a similar transduction efficiency in all target cells. Luciferase expression in transduced cells proved to be proportional to viral titer over a wide range (fig. S1D).

\section{Establishment of a SARS-CoV-2 cohort}

In parallel to the development of neutralization assays, a cohort was established in San Diego, California, of 17 donors who had previously been infected with SARS-CoV-2 (Fig. 2A, fig. S2A, and table S1). The cohort was $47 \%$ female and the average age was 50 years. Infection was determined by a positive SARS-CoV-2 PCR test from a nasopharyngeal swab. All donors also had symptoms consistent with COVID-19, and disease severity ranged from mild to severe, including intubation in one case, although all donors recovered. Donor plasma were tested for binding to recombinant SARS-CoV-2 and SARS-CoV-1 S and receptor binding domain (RBD) proteins, for binding to cell surface expressed spikes and for neutralization in both live replicating virus and pseudovirus assays (Fig. 2, B to D, and fig. S2B; 3 donors CC6, $\mathrm{CC} 12$ and $\mathrm{CC} 25$ that are further pursued below are highlighted).
Binding titers to SARS-CoV-2 S protein varied considerably, reaching $\mathrm{EC}_{50} \mathrm{~S}$ at serum dilutions of around $10^{4}$, with titers against the RBD about an order of magnitude less. Titers against SARS-CoV-1 $S$ protein were notably less than for SARS-CoV-2 $S$ protein and titers against SARS-CoV-1 RBD were only detected in a small number of donors. Neutralizing titers in the PSV assay varied over a wide range for SARSCoV-2 (Fig. 2D and fig. S2A) and were low or undetectable against SARS-CoV-1. Importantly, RBD binding and PSV neutralization were well correlated (Fig. 2E). There was also a positive correlation between cell surface spike binding and live replicating virus neutralization (fig. S2C). The titers in the PSV assay and the replicating virus assay were largely similar (figs. S2 and S3). In most later measurements, the PSV assay was preferred owing to its higher throughput.

\section{Antibody isolation and preliminary functional screens for downselection}

Cryopreserved PBMCs from eight donors were stained for memory B cells markers (CD19+/IgG+) and both Avi-tag biotinylated RBD and SARS-CoV-2 $\mathrm{S}$ antigen baits before singlecell sorting. S+ and S+/RBD+ memory B cells were present at an average frequency of $2.0 \%$ and $0.36 \%$, respectively, across the eight donors (fig. S4A). In total, 3160 antigen-positive $(\mathrm{Ag}+)$ memory B cells were sorted to rescue native heavy and light chain pairs for mAb production and validation (fig. S4B). A total of 2045 antibodies were cloned and expressed, which represents, on average, a $65 \%$ PCR recovery of paired variable genes and $>86 \%$ estimated recovery of fully functional cloned genes (fig. S4C). The bulk-transformed ligation products for both the heavy chain and light chain were transfected and tested for binding to RBD and S protein, and for neutralization in the SARS-CoV-2 pseudovirus assay using HeLa-ACE2 target cells (fig. S5).

The majority of transfected pairs resulted in IgG expression (92\%). Of these, $43 \%$ showed binding only to S protein while $5.9 \%$ bound to both $\mathrm{S}$ and RBD proteins and $0.1 \%$ bound only to RBD. The supernatants were also screened for binding to an unrelated HIV antigen (BG505 SOSIP) to eliminate non-specific or polyreactive supernatants. The supernatants were next evaluated for neutralization activity using SARS-CoV-2 and SARS-CoV-1 pseudoviruses. Strikingly, a small proportion of the binding antibodies showed neutralization activity and that activity was equally distributed between $\mathrm{RBD}+/ \mathrm{S}+$ and $\mathrm{S}+$ only binders despite a much larger number of S+ only binding supernatants as exemplified by the three donors $\mathrm{CC} 6, \mathrm{CC} 12$ and $\mathrm{CC} 25$, (Fig. 3A). These data indicate that viral infection generates a strong response against the non-RBD regions of S protein, but only a small proportion of that response is neutralizing. In contrast, there are fewer RBD binding antibodies but a larger proportion of these neutralize SARS-CoV-2 pseudovirus. Antibodies that 
tested positive for neutralization in the high-throughput screening were sequence confirmed and advanced for expression at large scale for additional characterization.

A total of 33 antibodies were prioritized for in depth characterization from the 3 donors, $\mathrm{CC} 6, \mathrm{CC} 12$ and $\mathrm{CC} 25$. Within that subset, we identified 25 distinct lineages, with 23 containing a single member (table S2). VH1 and VH3-gene families were notably prominent in these Abs and there was a diversity of CDR3 lengths (Fig. 3, B and C). There was one prominent example of a clonally expanded lineage, with 8 recovered clonal members that averaged $4.3 \%$ and $2.8 \%$ mutations from germline at the nucleotide level in the heavy chain and light chain, respectively (Fig. 3D). The remaining clones were relatively unmutated, averaging just above $1 \%$ mutation at the nucleotide level suggesting that these antibodies were primed by the ongoing COVID infection and likely not recalled from a previous endemic human coronavirus ( $\mathrm{HCoV}$ ) exposure. All antibodies that were expressed at scale were evaluated in standard ELISA-based polyreactivity assays with solubilized $\mathrm{CHO}$ membrane preparations, ssDNA and insulin $(7,8)$, and none were polyreactive (fig. S6).

\section{Functional activity of downselected antibodies}

The antibody hits that were identified in the high-throughput screening were next evaluated for epitope specificity by biolayer interferometry (BLI) using S and RBD proteins as capture antigens. The antigens were captured on anti-HIS biosensors before addition of saturating concentrations $(100 \mu \mathrm{g} / \mathrm{ml})$ of antibodies that were then followed by competing antibodies at a lower concentration $(25 \mu \mathrm{g} / \mathrm{ml})$. Accordingly, only antibodies that bind to a non-competing site would be detected in the assay. Among the antibodies evaluated, the results reveal three epitope bins for RBD (designated as RBD-A, RBD-B, and RBD-C) and three epitope bins for the S protein (designated as S-A, S-B, and S-C) (Fig. 4A and fig. S7). Interestingly, the $\mathrm{mAb} \mathrm{CC} 12.19$ appears to compete with antibodies targeting two different epitopes, RBD-B and S-A (fig. S7), which might indicate that this mAb targets an epitope spanning RBD-B and S-A. To evaluate epitope specificities further, we next assessed binding of the antibodies to extended RBD-constructs with subdomains (SD) 1 and 2 , including the independently folding RBD-SD1 and RBDSD1-2, and the N-terminal domain (NTD) (Fig. 4B and fig. S8, $\mathrm{A}$ and $\mathrm{B})$. None of the antibodies showed binding to the NTD. CC12.19 binds to all the other constructs, which supports the epitope binning data described in Fig. 4A. The other antibodies grouped in the S-A epitope bin that compete with $\mathrm{CC} 12.19$ show either no binding to RBD or RBD-SD constructs (CC12.20 and CC12.21) or do show binding to RBD-SD1 and RBD-SD1-2 but not RBD (CC12.23). These data suggest two competing epitopes within the S-A epitope bin; one that is confined to the non-RBD region of $\mathrm{S}$ protein and the other that includes some element of RBD-SD1-2. This interpretation will require further investigation by structural studies.

We next evaluated the mAbs for neutralization activity against SARS-CoV-2 and SARS-CoV-1 pseudoviruses. The neutralization $\mathrm{IC}_{50}$ potencies of these antibodies are shown in Fig. 4C and their associated maximum plateaus of neutralization (MPNs) are shown in Fig. 4D. A comparison of neutralization potencies between pseudovirus (fig. S8C) and live replicating virus (fig. S8D) is also included. Notably, the most potent neutralizing antibodies were those directed to RBD-A epitope including two antibodies, CC6.29 and CC6.30, that neutralize SARS-CoV-2 pseudovirus with an $\mathrm{IC}_{50}$ of $2 \mathrm{ng} / \mathrm{ml}$ and $1 \mathrm{ng} / \mathrm{ml}$, respectively (Fig. 4C). In comparison, antibodies directed to RBD-B tended to have higher $\mathrm{IC}_{50} \mathrm{~S}$ and many plateau below $100 \%$ neutralization. Despite this trend, CC6.33 is directed against RBD-B and showed complete neutralization of SARS-CoV-2 with an $\mathrm{IC}_{50}$ of $39 \mathrm{ng} / \mathrm{ml}$ and also neutralized SARS-CoV-1 with an IC50 of $162 \mathrm{ng} / \mathrm{ml}$. This was the only antibody that showed potent neutralization of both pseudoviruses. The antibodies that do not bind to RBD and are directed to non-RBD epitopes on S protein all show poor neutralization potencies and MPNs well below $100 \%$.

To evaluate whether the RBD-A epitope might span the ACE2 binding site, we next performed cell surface competition experiments. Briefly, antibodies were premixed with biotinylated S (Fig. 4E) or RBD (Fig. 4F) proteins at a molar ratio of 4:1 of antibodies to target antigen. The mixture was then incubated with the HeLa-ACE2 cell line and the percent competition against ACE2 receptor was recorded by comparing percent binding of the target antigen with and without antibody present (fig. S8E). The antibodies targeting the RBD-A epitope compete best against the ACE2 receptor and the neutralization $\mathrm{IC}_{50}$ correlates well with the percent competition for ACE2 receptor binding for both S protein (Fig. 4E) and for RBD (Fig. 4F). We also assessed the affinity of all RBD-specific antibodies to soluble RBD by surface plasmon resonance (SPR) and found a poor correlation between affinity and neutralization potency (Fig. 4G and fig. S9). However, the correlation is higher when limited to antibodies targeting the RBD-A epitope. The lack of a correlation between RBD binding and neutralization for mAbs contrasts with the strong correlation described earlier for serum RBD binding and neutralization. Overall, the data highlight epitope RBDA as the preferred target for eliciting neutralizing antibodies and that corresponding increases in affinity of mAbs to RBDA will likely result in corresponding increases in neutralization potency.

SARS-CoV-2 has shown some propensity for mutation as it has circulated worldwide as evidenced for example in the emergence of the D614G variant (9). We investigated the activity of 5 nAbs against 6 viral variants that have been reported. The 3 sera studied above neutralized all the variants 
(fig. S10A). All 5 nAbs neutralized the D614G variant. However, one variant with a mutation in the ACE2 binding site (G476S) did show effectively complete resistance to one of the nAbs and another variant (V367F) showed a 10-fold higher $\mathrm{IC}_{50}$ than the WA-1 strain (fig. S10B).

\section{Passive transfer of neutralizing antibodies and SARS- CoV-2 challenge in Syrian hamsters}

To investigate the relationship between in vitro neutralization and protection in vivo against SARS-CoV-2, we selected two mAbs for passive transfer/challenge experiments in a Syrian hamster animal model based on a summary of the $\mathrm{nAb}$ data (table S3 and fig. S11). The experimental design for the passive transfer study is shown in Fig. 5A. In the first experiment, we tested $\mathrm{nAb} \mathrm{CC} 12.1$, which targets the RBD-A epitope and has an in vitro $\mathrm{IC}_{50}$ neutralization of $0.019 \mu \mathrm{g} / \mathrm{ml}$ against pseudovirus and in the second we tested $\mathrm{nAb} C 12.23$, which targets the S-B epitope with an $\mathrm{IC}_{50}$ neutralization of $22 \mu \mathrm{g} / \mathrm{ml}$. In both experiments an unrelated antibody to dengue virus, Den3, was used as a control. The anti-SARS-CoV-2 nAbs were delivered at 5 different concentrations to evaluate dose-dependent protection starting at $2 \mathrm{mg}$ /animal (average of $16.5 \mathrm{mg} / \mathrm{kg}$ ) at the highest dose and $8 \mu \mathrm{g} /$ animal at the lowest dose. The Den3 control antibody was delivered at a single dose of $2 \mathrm{mg}$ /animal. Sera were collected from each animal 12 hours post IP infusion of the antibody and all animals were subsequently challenged with a dose of $1 \times 10^{6} \mathrm{PFU}$ of SARSCoV-2 (USA-WA1/2020) by intranasal administration 12 hours post antibody infusion (Fig. 5A).

Syrian hamsters typically clear virus within one week after SARS-CoV-1 infection (10). Accordingly, the hamsters were weighed as a measure of disease due to infection. Lung tissues were collected to measure viral load on day 5 . A data summary is presented in Fig. 5B and fig. S12A for animals that received $\mathrm{CC} 12.1$, which targets the RBD-A epitope. The control animals that received Den3 lost on average $13.6 \%$ of body weight at 5 days post virus challenge. In comparison, the animals that received the neutralizing RBD-A antibody at a dose of $2 \mathrm{mg}$ (average of $16.5 \mathrm{mg} / \mathrm{kg}$ ) or $500 \mu \mathrm{g}$ (average of $4.2 \mathrm{mg} / \mathrm{kg}$ ) exhibited no weight loss. However, animals that received a dose of $125 \mu \mathrm{g}$ (average of $0.9 \mathrm{mg} / \mathrm{kg}$ ) had an average $8 \%$ loss of body weight, while animals that received a dose of $31 \mu \mathrm{g} / \mathrm{ml}(0.2 \mathrm{mg} / \mathrm{kg})$ and $8 \mu \mathrm{g} / \mathrm{ml}(0.06 \mathrm{mg} / \mathrm{kg})$ lost $15.8 \%$ and $16.7 \%$ of body weight, respectively. We note these animals showed a trend for greater weight loss than control animals but this did not achieve statistical significance (table S4). Given concerns about antibody-mediated enhanced disease in SARS-CoV-2 infection, this observation merits further attention using larger animal group sizes. The weight loss data are further corroborated by quantification of lung viral load measured by real-time PCR (Fig. 5C) and showed a moderate correlation to weight loss. The data indicate comparable viral loads between the three higher doses $(2 \mathrm{mg}$, $500 \mu \mathrm{g}$, and $125 \mu \mathrm{g}$ ) of nAbs. In contrast, equivalent viral loads were observed between the control group receiving Den3 and the low dose groups receiving $31 \mu \mathrm{g}$ and $8 \mu \mathrm{g}$ of nAb. In contrast to the $\mathrm{nAb}$ to RBD-A, the less potent and incompletely neutralizing antibody to the S-B epitope showed no evidence of protection at any concentration compared to the control animals (fig. S12B).

To determine the antibody serum concentrations that may be required for protection against disease from SARSCoV-2 infection, we also measured the antibody serum concentrations just prior to intranasal virus challenge (Fig. 5D). The data highlight that an antibody serum concentration of approximately $22 \mu \mathrm{g} / \mathrm{ml}$ of nAb (1160 x PSV neutralization $\mathrm{IC}_{50}$ ) enables full protection and a serum concentration of 12 $\mu \mathrm{g} / \mathrm{ml}\left(630 \times \mathrm{PSV}\right.$ neutralization $\mathrm{IC}_{50}$ ) is adequate for $50 \%$ reduced disease as measured by weight loss. The effective antibody concentration required at the site of infection to protect from disease remains to be determined. Sterilizing immunity at serum concentrations that represent a large multiplier of the in vitro neutralizing $\mathrm{IC}_{50}$ is observed for many viruses (11).

\section{Discussion}

Using a high-throughput rapid system for antibody discovery, we isolated more than $1000 \mathrm{mAbs}$ from 3 convalescent donors by memory B cell selection using SARS-CoV-2 S or RBD recombinant proteins. About half of the mAbs isolated could be expressed and also bind effectively to either S and/or RBD proteins. Only a small fraction of these Abs was neutralizing, highlighting the value of deep mining of responses to access the most potent Abs (4).

A range of nAbs were isolated to different sites on the $S$ protein. The most potent Abs, reaching single digit $\mathrm{ng} / \mathrm{ml}$ $\mathrm{IC}_{50} \mathrm{~S}$ in PSV assays, are targeted to a site that, judged by competition studies, overlaps the ACE2 binding site. Only one of the Abs, directed to RBD-B, neutralized SARS-CoV-1 PSV, as may be anticipated given the differences in ACE2 contact residues between the two viruses (fig. S13) and given that the selections were performed with SARS-CoV-2 target proteins. Abs directed to the RBD but not competitive with soluble ACE2, (although they may be competitive in terms of an array of membrane-bound ACE2 molecules interacting with an array of spike proteins on a virion), are generally less potent neutralizers and tend to show incomplete neutralization, plateauing at around or less than $50 \%$ neutralization. The one exception is the cross-reactive RBD-B antibody above. Similar lower potency and incomplete neutralization are observed for Abs to the S protein that are not reactive with recombinant RBD. The cause(s) of these incomplete neutralization phenomena is unclear but presumably originates in some spike protein heterogeneity, either glycan, cleavage or conformationally based. In any case, the RBD-A nAbs that directly 
compete with ACE2 are clearly the most preferred for prophylactic and therapeutic applications, and as reagents to define $\mathrm{nAb}$ epitopes for vaccine design. We note that, even for a small sampling of naturally occurring viral variants, two were identified that showed notable resistance to individual potent nAbs to the WA-1 strain and neutralization resistance will need to be considered in planning for clinical applications of nAbs. Cocktails of nAbs may be required.

In terms of nAbs as passive reagents, the efficacy of a potent anti-RBD nAb in vivo in Syrian hamsters is promising in view of the positive attributes of this animal model (12) and suggests that human studies are merited. Nevertheless, as for any animal model, there are many limitations, including, in the context of antibody protection, differences in effector cells and $\mathrm{Fc}$ receptors between humans and hamsters. The failure of the non-RBD S-protein nAb to protect in the animal model is consistent with its lower potency and, likely most importantly, its inability to fully neutralize challenge virus. In the context of human studies, improved potency of protective nAbs by enhancing binding affinity to the RBD epitope identified, improved half-life and reduced Fc receptor binding to minimize potential antibody dependent enhancement (ADE) effects, should they be identified as concerning, are all antibody engineering goals to be considered. As observed for heterologous B cell responses against different serotypes of flavivirus infection, there is a possibility, but no current experimental evidence, that subtherapeutic vaccine serum responses or subtherapeutic nAb titers could potentially exacerbate future coronavirus infection disease burden by expanding the viral replication and/or cell tropism of the virus. If ADE is found for SARS-CoV-2 and operates at sub-neutralizing concentrations of neutralizing antibodies as it can for dengue virus (13) then it would be important, from a vaccine standpoint, to carefully define the full range of $n A b$ epitopes on the S protein as we have begun here. From a passive antibody standpoint, it would be important to maintain high $\mathrm{nAb}$ concentrations or appropriately engineer nAbs.

The nAbs described have remarkably little SHM, typically one or two mutations in the $\mathrm{VH}$ gene and one or two in the VL gene. Such low SHM may be associated with the isolation of the nAbs relatively soon after infection, and perhaps before affinity-maturation has progressed. Low SHM has also been described for potent nAbs to Ebola virus, respiratory syncytial virus (RSV), Middle East Respiratory Syndrome coronavirus (MERS-CoV) and yellow fever virus (14-17) and may indicate that the human naïve repertoire is often sufficiently diverse to respond effectively to many pathogens with little mutation. Of course, nAb efficacy and titer may increase over time as described for other viruses and it will be interesting to see if even more potent nAbs to SARS-CoV-2 evolve in our donors in the future.
What do our results suggest for SARS-CoV-2 vaccine design? In the first instance, the results suggest a focus on the RBD and indeed strong $\mathrm{nAb}$ responses have been described by immunizing mice with a multivalent presentation of RBD (18). The strong preponderance of non-neutralizing antibodies and very few nAbs to $S$ protein that we isolated could arise for a number of reasons including: (i) the recombinant $\mathrm{S}$ protein that we used to select $B$ cells is a poor representation of the native spike on virions. In other words, there may be many nAbs to $\mathrm{S}$ but we failed to isolate them because of the selecting antigen, (ii) the recombinant $S$ protein that we used is close to native but non-neutralizing antibodies bind to sites on $\mathrm{S}$ that do not interfere with viral entry, (iii) the $\mathrm{S}$ protein in natural infection disassembles readily generating a strong $\mathrm{Ab}$ response to "viral debris" that is non-neutralizing because the antibodies recognize protein surfaces that are not exposed on the native spike. Importantly, the availability of both neutralizing and non-neutralizing antibodies generated in this study will facilitate evaluation of S protein immunogens for presentation of neutralizing and non-neutralizing epitopes and promote effective vaccine design. The design of an immunogen that improves on the quality of nAbs elicited by natural infection may well emerge as an important goal of vaccine efforts (19).

In summary, we describe the very rapid generation of neutralizing antibodies to a newly emerged pathogen. The antibodies can find clinical application and will aid in vaccine design.

\section{REFERENCES AND NOTES}

1. The IMpact-RSV Study Group, Palivizumab, a humanized respiratory syncytial virus monoclonal antibody, reduces hospitalization from respiratory syncytial virus infection in high-risk infants. Pediatrics 102, 531-537 (1998). doi:10.1542/peds.102.3.531

2. D. Corti, J. Misasi, S. Mulangu, D. A. Stanley, M. Kanekiyo, S. Wollen, A. Ploquin, N. A. Doria-Rose, R. P. Staupe, M. Bailey, W. Shi, M. Choe, H. Marcus, E. A. Thompson, A. Cagigi, C. Silacci, B. Fernandez-Rodriguez, L. Perez, F. Sallusto, F. Vanzetta, G. Agatic, E. Cameroni, N. Kisalu, I. Gordon, J. E. Ledgerwood, J. R. Mascola, B. S. Graham, J.-J. Muyembe-Tamfun, J. C. Trefry, A. Lanzavecchia, N. J. Sullivan, Protective monotherapy against lethal Ebola virus infection by a potently neutralizing antibody. Science 351, 1339-1342 (2016). doi:10.1126/science.aad5224 Medline

3. J. Misasi, M. S. A. Gilman, M. Kanekiyo, M. Gui, A. Cagigi, S. Mulangu, D. Corti, J. E. Ledgerwood, A. Lanzavecchia, J. Cunningham, J. J. Muyembe-Tamfun, U. Baxa, B. S. Graham, Y. Xiang, N. J. Sullivan, J. S. McLellan, Structural and molecular basis for Ebola virus neutralization by protective human antibodies. Science 351, 13431346 (2016). doi:10.1126/science.aad6117 Medline

4. L. M. Walker, D. R. Burton, Passive immunotherapy of viral infections: 'superantibodies' enter the fray. Nat. Rev. Immunol. 18, 297-308 (2018). doi:10.1038/nri.2017.148 Medline

5. T. Giroglou, J. Cinatl Jr., H. Rabenau, C. Drosten, H. Schwalbe, H. W. Doerr, D. von Laer, Retroviral vectors pseudotyped with severe acute respiratory syndrome coronavirus S protein. J. Virol. 78, 9007-9015 (2004). doi:10.1128/JVL.78.17.9007-9015.2004 Medline

6. C. E. McBride, J. Li, C. E. Machamer, The cytoplasmic tail of the severe acute respiratory syndrome coronavirus spike protein contains a novel endoplasmic reticulum retrieval signal that binds COPI and promotes interaction with membrane protein. J. Virol. 81, 2418-2428 (2007). doi:10.1128/JVL.02146-06 $\underline{\text { Medline }}$ 
7. T. Jain, T. Sun, S. Durand, A. Hall, N. R. Houston, J. H. Nett, B. Sharkey, B. Bobrowicz, I. Caffry, Y. Yu, Y. Cao, H. Lynaugh, M. Brown, H. Baruah, L. T. Gray, E. M. Krauland, Y. Xu, M. Vásquez, K. D. Wittrup, Biophysical properties of the clinical-stage antibody landscape. Proc. Natl. Acad. Sci. U.S.A. 114, $944-949$ (2017). doi:10.1073/pnas.1616408114 Medline

8. J. G. Jardine, D. Sok, J.-P. Julien, B. Briney, A. Sarkar, C.-H. Liang, E. M. Scherer, C. J. Henry Dunand, Y. Adachi, D. Diwanji, J. Hsueh, M. Jones, O. Kalyuzhniy, M. Kubitz, S. Spencer, M. Pauthner, K. L. Saye-Francisco, F. Sesterhenn, P. C. Wilson, D. A. Galloway, R. L. Stanfield, I. A. Wilson, D. R. Burton, W. R. Schief, Minimally mutated HIV-1 broadly neutralizing antibodies to guide reductionist vaccine design. PLOS Pathog. 12, el005815 (2016). doi:10.1371/journal.ppat.1005815 Medline

CoV-Glue, Amino acid replacements; http://cov-

. A. Roberts, L. Vogel, J. Guarner, N. Hayes, B. Murphy, S. Zaki, K. Subbarao, Severe acute respiratory syndrome coronavirus infection of golden Syrian hamsters. J. Virol. 79, 503-511 (2005). doi:10.1128/JVI.79.1.503-511.2005 Medline

11. P. W. Parren, D. R. Burton, The antiviral activity of antibodies in vitro and in vivo. Adv. Immunol. 77, 195-262 (2001). doi:10.1016/S0065-2776(01)77018-6 Medline

12. J. F.-W. Chan, A. J. Zhang, S. Yuan, V. K.-M. Poon, C. C.-S. Chan, A. C.-Y. Lee, W.M. Chan, Z. Fan, H.-W. Tsoi, L. Wen, R. Liang, J. Cao, Y. Chen, K. Tang, C. Luo, J.P. Cai, K.-H. Kok, H. Chu, K.-H. Chan, S. Sridhar, Z. Chen, H. Chen, K. K.-W. To, K.Y. Yuen, Simulation of the clinical and pathological manifestations of Coronavirus Disease 2019 (COVID-19) in golden Syrian hamster model: Implications for disease pathogenesis and transmissibility. Clin. Infect. Dis. ciaa325 (2020). doi:10.1093/cid/ciaa325 Medline

13. S. B. Halstead, Dengue antibody-dependent enhancement: Knowns and unknowns. Microbiol. Spectr. 2, (2014). doi:10.1128/microbiolspec.AID-00222014 Medline

14. C. W. Davis, K. J. L. Jackson, A. K. McElroy, P. Halfmann, J. Huang, C. Chennareddy, A. E. Piper, Y. Leung, C. G. Albariño, I. Crozier, A. H. Ellebedy, J. Sidney, A. Sette, T. Yu, S. C. A. Nielsen, A. J. Goff, C. F. Spiropoulou, E. O. Saphire, G. Cavet, Y. Kawaoka, A. K. Mehta, P. J. Glass, S. D. Boyd, R. Ahmed, Longitudinal analysis of the human B cell response to Ebola virus infection. Cell 177, 1566-1582.e17 (2019). doi:10.1016/i.cell.2019.04.036 Medline

15. T. Ying, P. Prabakaran, L. Du, W. Shi, Y. Feng, Y. Wang, L. Wang, W. Li, S. Jiang, D. S. Dimitrov, T. Zhou, Junctional and allele-specific residues are critical for MERSCoV neutralization by an exceptionally potent germline-like antibody. Nat. Commun. 6, 8223 (2015). doi:10.1038/ncomms9223 Medline

16. E. Goodwin, M. S. A. Gilman, D. Wrapp, M. Chen, J. O. Ngwuta, S. M. Moin, P. Bai, A. Sivasubramanian, R. I. Connor, P. F. Wright, B. S. Graham, J. S. McLellan, L. M. Walker, Infants infected with Respiratory Syncytial Virus generate potent neutralizing antibodies that lack somatic hypermutation. Immunity 48, 339349.e5 (2018). doi:10.1016/i.immuni.2018.01.005 Medline

17. A. Z. Wec, D. Haslwanter, Y. N. Abdiche, L. Shehata, N. Pedreño-Lopez, C. L. Moyer, Z. A. Bornholdt, A. Lilov, J. H. Nett, R. K. Jangra, M. Brown, D. I. Watkins, C. Ahlm, M. N. Forsell, F. A. Rey, G. Barba-Spaeth, K. Chandran, L. M. Walker, Longitudinal dynamics of the human B cell response to the yellow fever 17D vaccine. Proc. Natl. Acad. Sci. U.S.A. 117, 6675-6685 (2020). doi:10.1073/pnas.1921388117 Medline

18. B. D. Quinlan, H. Mou, L. Zhang, Y. Guo, W. He, A. Ojha, M. S. Parcells, G. Luo, W. Li, G. Zhong, H. Choe, M. Farzan, The SARS-CoV-2 receptor-binding domain elicits a potent neutralizing response without antibody-dependent enhancement. Microbiology e0003749 (2020).

19. D. R. Burton, L. M. Walker, Rational vaccine design in the time of COVID-19. Cell Host Microbe 27, 695-698 (2020). doi:10.1016/j.chom.2020.04.022 Medline

20. R. N. Kirchdoerfer, C. A. Cottrell, N. Wang, J. Pallesen, H. M. Yassine, H. L. Turner, K. S. Corbett, B. S. Graham, J. S. McLellan, A. B. Ward, Pre-fusion structure of a human coronavirus spike protein. Nature 531, 118-121 (2016). doi:10.1038/nature17200 Medline

21. D. Wrapp, N. Wang, K. S. Corbett, J. A. Goldsmith, C.-L. Hsieh, O. Abiona, B. S. Graham, J. S. McLellan, Cryo-EM structure of the 2019-nCoV spike in the prefusion conformation. Science 367, 1260-1263 (2020). doi:10.1126/science.abb2507 Medline
22. L. M. Walker, S. K. Phogat, P.-Y. Chan-Hui, D. Wagner, P. Phung, J. L. Goss, T. Wrin, M. D. Simek, S. Fling, J. L. Mitcham, J. K. Lehrman, F. H. Priddy, O. A. Olsen, S. M. Frey, P. W. Hammond, S. Kaminsky, T. Zamb, M. Moyle, W. C. Koff, P. Poignard, D. R. Burton; Protocol G Principal Investigators, Broad and potent neutralizing antibodies from an African donor reveal a new HIV-1 vaccine target. Science 326 285-289 (2009). doi:10.1126/science.1178746 Medline

23. X. Wu, Z.-Y. Yang, Y. Li, C.-M. Hogerkorp, W. R. Schief, M. S. Seaman, T. Zhou, S. D. Schmidt, L. Wu, L. Xu, N. S. Longo, K. McKee, S. O'Dell, M. K. Louder, D. L. Wycuff, Y. Feng, M. Nason, N. Doria-Rose, M. Connors, P. D. Kwong, M. Roederer, R. T. Wyatt, G. J. Nabel, J. R. Mascola, Rational design of envelope identifies broadly neutralizing human monoclonal antibodies to HIV-1. Science 329, 856861 (2010). doi:10.1126/science.1187659 Medline

24. D. Sok, M. J. van Gils, M. Pauthner, J.-P. Julien, K. L. Saye-Francisco, J. Hsueh, B. Briney, J. H. Lee, K. M. Le, P. S. Lee, Y. Hua, M. S. Seaman, J. P. Moore, A. B. Ward, I. A. Wilson, R. W. Sanders, D. R. Burton, Recombinant HIV envelope trimer selects for quaternary-dependent antibodies targeting the trimer apex. Proc. Natl. Acad. Sci. U.S.A. 111, 17624-17629 (2014). doi:10.1073/pnas.1415789111 Medline

25. T. Tiller, E. Meffre, S. Yurasov, M. Tsuiji, M. C. Nussenzweig, H. Wardemann, Efficient generation of monoclonal antibodies from single human $B$ cells by single cell RT-PCR and expression vector cloning. J. Immunol. Methods 329, 112-124 (2008). doi:10.1016/i.jim.2007.09.017 Medline

26. N. A. Doria-Rose, C. A. Schramm, J. Gorman, P. L. Moore, J. N. Bhiman, B. J. DeKosky, M. J. Ernandes, I. S. Georgiev, H. J. Kim, M. Pancera, R. P. Staupe, H. R. Altae-Tran, R. T. Bailer, E. T. Crooks, A. Cupo, A. Druz, N. J. Garrett, K. H. Hoi, R. Kong, M. K. Louder, N. S. Longo, K. McKee, M. Nonyane, S. O'Dell, R. S. Roark, R. S. Rudicell, S. D. Schmidt, D. J. Sheward, C. Soto, C. K. Wibmer, Y. Yang, Z. Zhang, J. C. Mullikin, J. M. Binley, R. W. Sanders, I. A. Wilson, J. P. Moore, A. B. Ward, G. Georgiou, C. Williamson, S. S. Abdool Karim, L. Morris, P. D. Kwong, L. Shapiro, J. R. Mascola; NISC Comparative Sequencing Program, Developmental pathway for potent V1V2-directed HIV-neutralizing antibodies. Nature 509, 55-62 (2014). doi:10.1038/nature13036 Medline

27. M.-P. Lefranc, V. Giudicelli, C. Ginestoux, J. Jabado-Michaloud, G. Folch, F. Bellahcene, Y. Wu, E. Gemrot, X. Brochet, J. Lane, L. Regnier, F. Ehrenmann, G. Lefranc, P. Duroux, IMGT, the international ImMunoGeneTics information system. Nucleic Acids Res. 37, D1006-D1012 (2009). doi:10.1093/nar/gkn838 Medline

\section{ACKNOWLEDGMENTS}

We thank Tom Gilman, Andrea Salazar and Biosero for their contribution to highthroughput pipeline generation. We also thank Barney Graham and Jason S. McLellan for provision of materials. We thank Laura Walker for valuable manuscript discussions. We thank all the COVID-19 cohort participants for donating samples. Funding: This work was supported by the NIH CHAVD (UM1 Al44462 to I.A.W., B.B., D.S. and D.R.B.), R01 (Al132317, Al073148 to D.N.) and K99 (Al145762 to R.K.A.) awards, the IAVI Neutralizing Antibody Center, the Bill and Melinda Gates Foundation (OPP 1170236 to I.A.W. and D.R.B.), (OPP 1183956 to J.E.V.) and (OPP1196345/ INV-008813 to DS and DRB). This work was also supported by the John and Mary Tu Foundation and the Pendleton Trust. Author contributions: T.F.R., E.L., D.S, J.G.J and D.R.B. conceived and designed the study. T.F.R., N.B., E.G., M.P., S.R., S.A.R. and D.M.S. recruited donors, collected and processed plasma and PBMC samples. F.Z., A. B., L.Y., M.P., and G.S. performed ELISA and cell binding assays. F.Z, D.H, N.B., L.Y., M.P., L.P., and J.R. developed and performed neutralization experiments. F.Z., A.B., O.L., C.S., J.W., R.K.A., E.L., D.S., J.G.J. performed antibody isolation, cloning, expression and competition experiments. W-T.H., G.S., S.C., R.A., N.C.W., M.Y. and I.A.W. generated recombinant antigens. T.F.R., L.Y. and M.J.R., and J.R.T. carried out animal studies and Q-PCR viral load measurements. T.F.R., F.Z., D.H., N.B., G.S., J.W., J.H., M.J.R., D.N., J.R.T., R.A., B.B., E.L., D.S., J.G.J. and D.R.B. designed experiments and analyzed the data. E.L., D.S., J.G.J and D.R.B. wrote the paper and all authors reviewed and edited the paper. Competing interests: D.R.B., D.H., J.G.J., E.L., T.F.R., D.S. and F.Z. are listed as inventors on pending patent applications describing the SARS-CoV-2 antibodies. D.R.B. is a consultant for IAVI. All other authors have no competing interests to declare. Data and materials availability: Antibody sequences have been deposited in GenBank under accession numbers MT594038-MT594103. Antibody plasmids are available from Dennis Burton under an MTA from The Scripps Research Institute. 
This work is licensed under a Creative Commons Attribution 4.0 International

(CC BY 4.0) license, which permits unrestricted use, distribution, and

reproduction in any medium, provided the original work is properly cited. To view

a copy of this license, visit https://creativecommons.org/licenses/by/4.0/.

This license does not apply to figures/photos/artwork or other content included in the article that is credited to a third party; obtain authorization from the rights holder before using such material.

\section{SUPPLEMENTARY MATERIALS}

science.sciencemag.org/cgi/content/full/science.abc7520/DC1

Materials and Methods

Figs. S1 to S13

Tables S1 to S4

References (20-27)

MDAR Reproducibility Checklist

12 May 2020; accepted 11 June 2020

Published online 15 June 2020

10.1126/science.abc7520 


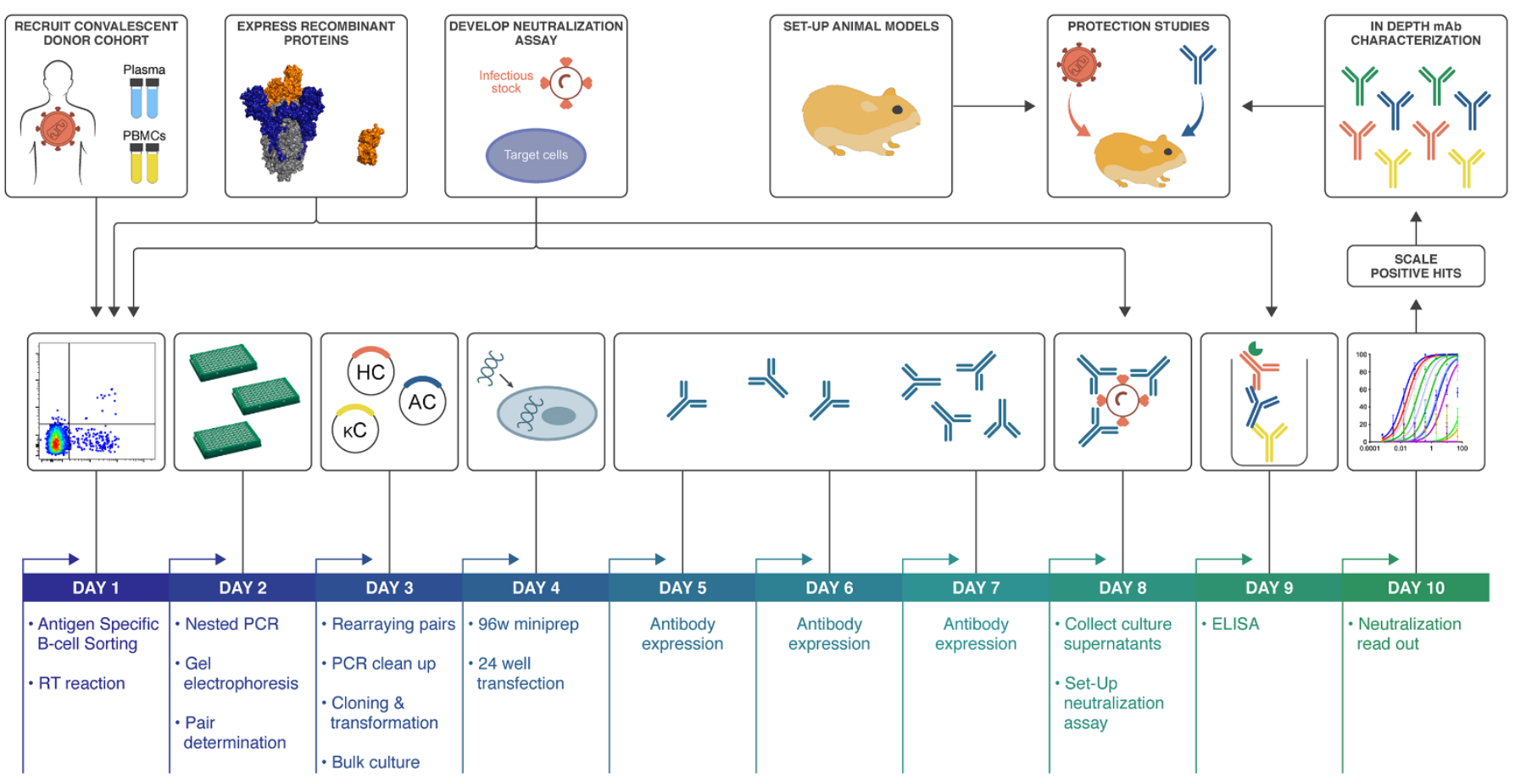

Fig. 1. SARS-CoV-2 neutralizing antibody isolation strategy. A natural infection cohort was established to collect plasma and PBMC samples from individuals who recovered from COVID-19. In parallel, functional assays were developed to rapidly screen plasma samples for SARS-CoV-2 neutralizing activity. SARS-CoV-2 recombinant surface proteins were also produced to use as baits in single memory B cell sorting and downstream functional characterization of isolated mAbs. Finally, a Syrian hamster animal model was set-up to evaluate mAb passive immunization and protection. The standard mAb isolation pipeline was optimized to facilitate high-throughput amplification, cloning, expression and functional screening of hundreds of unpurified $\mathrm{Ab}$ heavy and light chain pairs isolated from each of several selected neutralizers in only 10 days. Selected pairs were scaled-up to purify IgG for validation and characterization experiments. Potent neutralizing mAbs were selected to evaluate protection in the Syrian hamster model. 
A

\begin{tabular}{ll}
\hline Patient Demographics & \\
\hline Participants & 17 \\
\hline Female & $8(47 \%)$ \\
Male & $9(53 \%)$ \\
\hline Average Age & 50 \\
\hline Days post-symptom onset & 17 \\
\hline Disease severity & \\
\hline Mild & $10(58 \%)$ \\
Moderate & $3(18 \%)$ \\
Severe & $3(18 \%)$ \\
Critical (intubated) & $1(6 \%)$ \\
\hline Significant past medical history & \\
\hline None & $13(76 \%)$ \\
Asthma & 1 \\
Hypertension & 1 \\
HIV & 1 \\
Chronic kidney disease & 1 \\
Coronary artery disease & 1 \\
Other & 1 \\
\hline
\end{tabular}

D

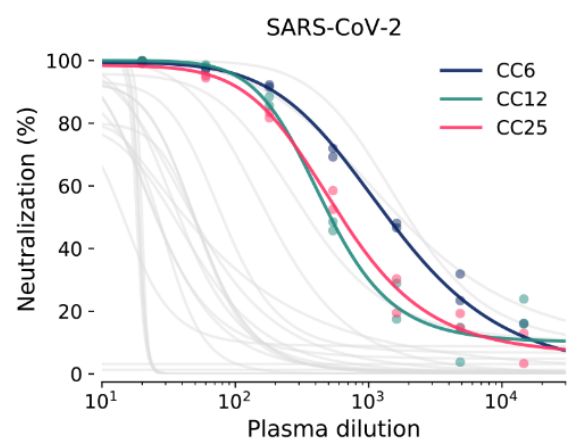

B
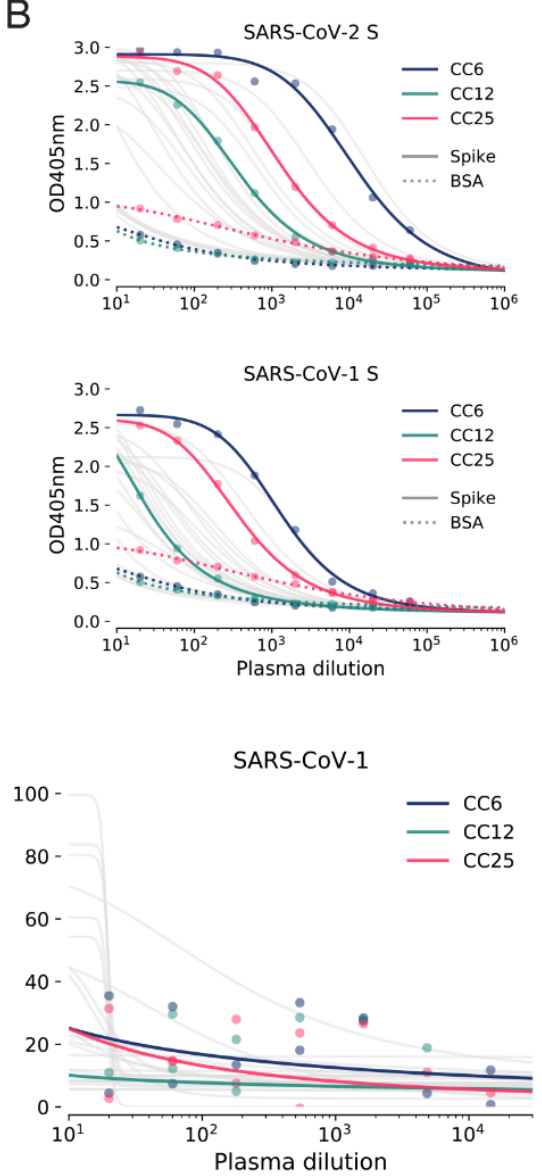

C
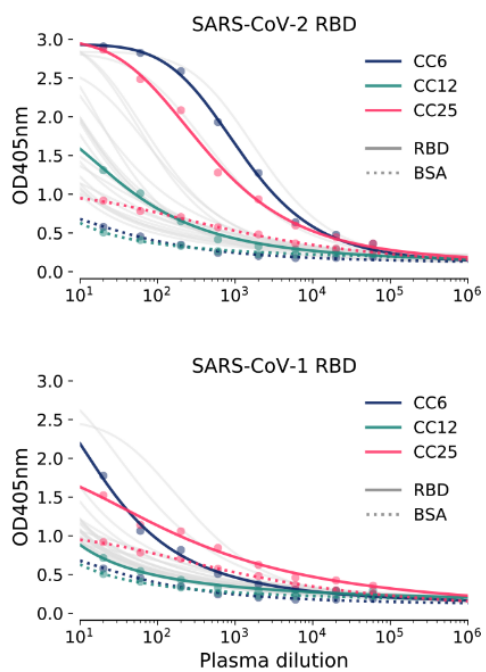

E

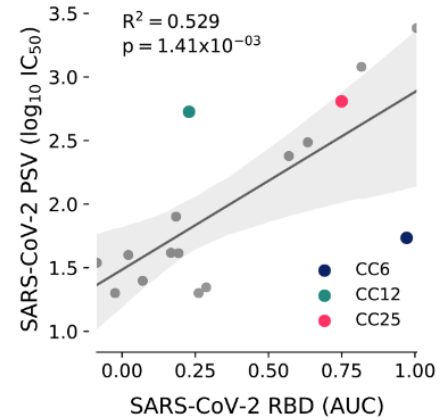

Fig. 2. COVID-19 cohort functional screening. (A) Demographics of the UCSD COVID-19 cohort (CC) participants. CC plasma were tested for binding to SARS-CoV-1 and SARS-CoV-2 S proteins (B) and RBD subunits $(C)$ by ELISA. Background binding of plasma to BSA-coated plates is represented by a dashed line.

(D) Plasma were also tested for neutralization of pseudotyped (PSV) SARS-CoV-1 and SARS-CoV-2 virions.

(E) Correlation between PSV SARS-CoV-2 neutralization and RBD subunit ELISA binding area-under-the-curve (AUC). AUC was computed using Simpson's rule. The 95\% confidence interval of the regression line is shown in light grey and was estimated by performing 1,000 bootstrap re-samplings. $R^{2}$ and $p$ values of the regression are also indicated. CC participants from whom mAbs were isolated are specifically highlighted in dark blue (CC6), pine green (CC12) and hot pink (CC25). 
A
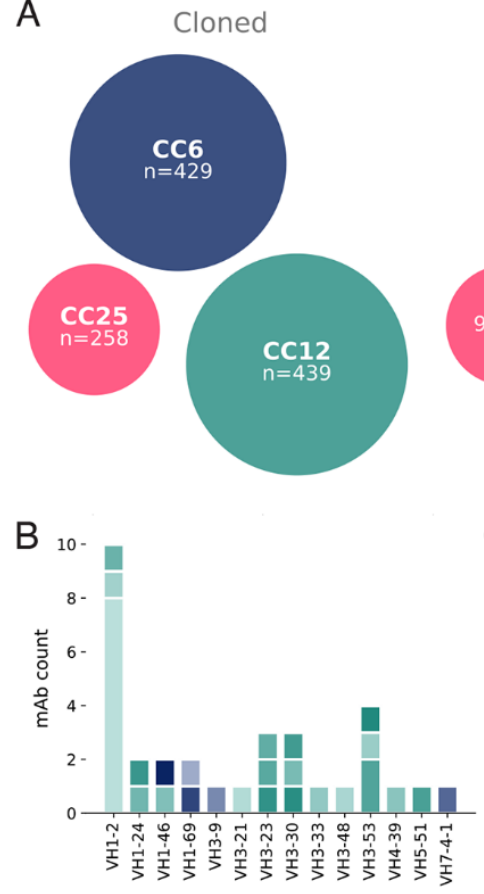

Expressed
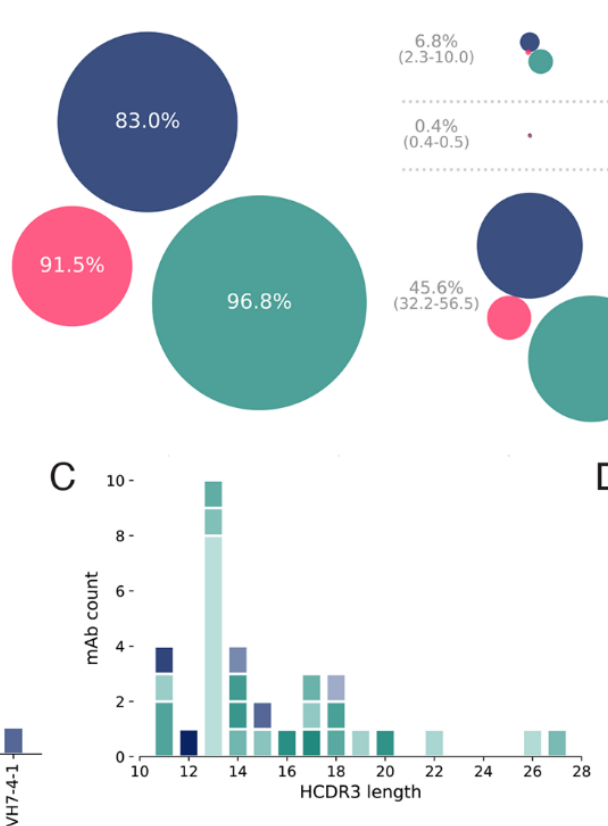

Binders

Neutralizers

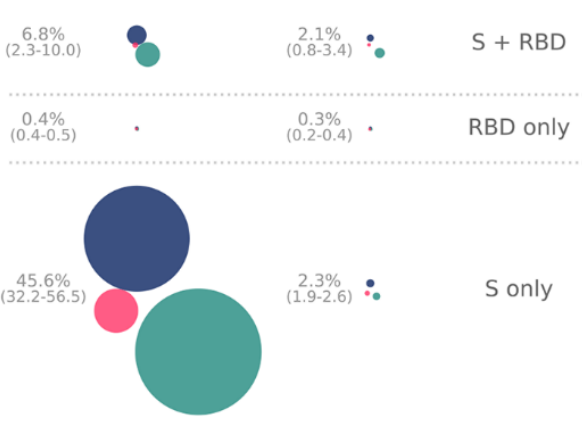

D

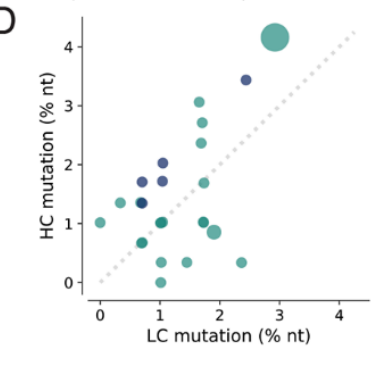

Fig. 3. Antibody isolation and functional screening for SARS-CoV antigen binding and neutralization. (A) Antibody downselection process from 3 donors, presented as bubble plots. The areas of the bubbles for each donor are sized, based on the number of antibodies that were cloned and transfected, then scaled according to the number that were positive in subsequent assays. All antibodies that expressed at measurable levels were tested for binding to $S$ protein and RBD to determine their specificity, and then screened for neutralization. (B) $\mathrm{VH}$ gene distribution of downselected mAbs. Antibodies are colored by their respective clonal lineages. (C) Heavy chain CDR3 lengths of downselected mAbs. Antibodies are colored by their respective clonal lineages. (D) Mutation frequency of downselected $\mathrm{mAb}$ lineages. Bubble position represents the mean mutation frequency for each lineage, with bubble area proportional to the lineage size. 
A

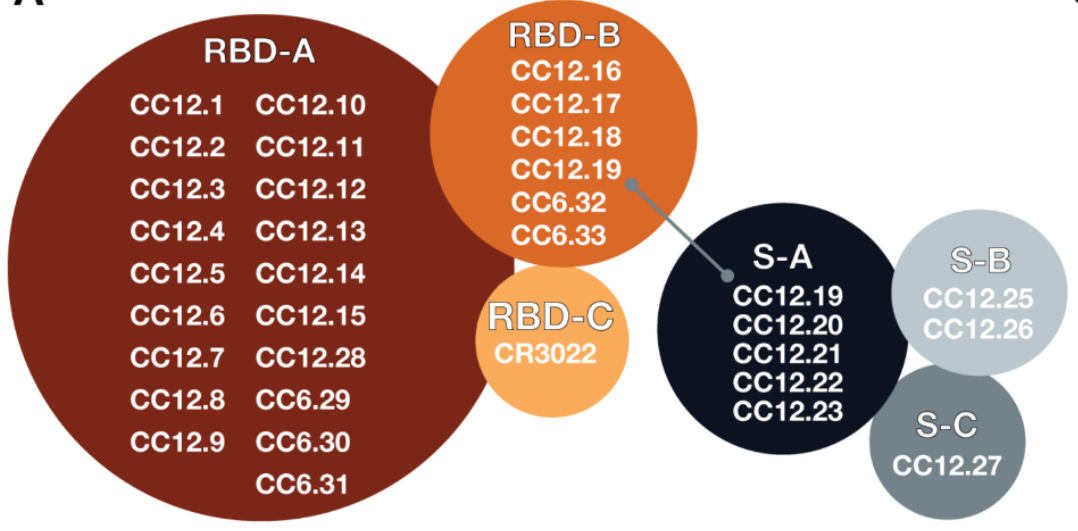

B ${ }_{10}^{2} 7 \quad$ Antibody binding to target antigens D

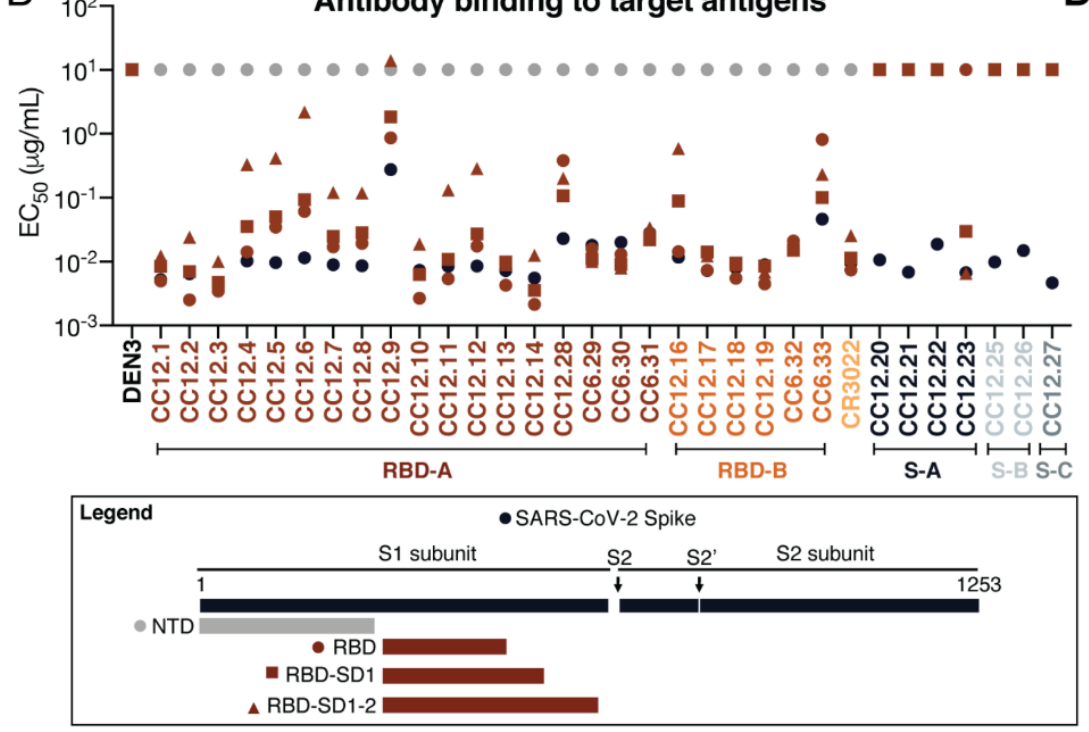

E

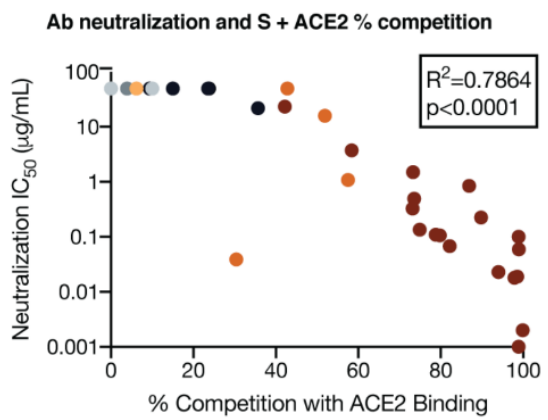

C
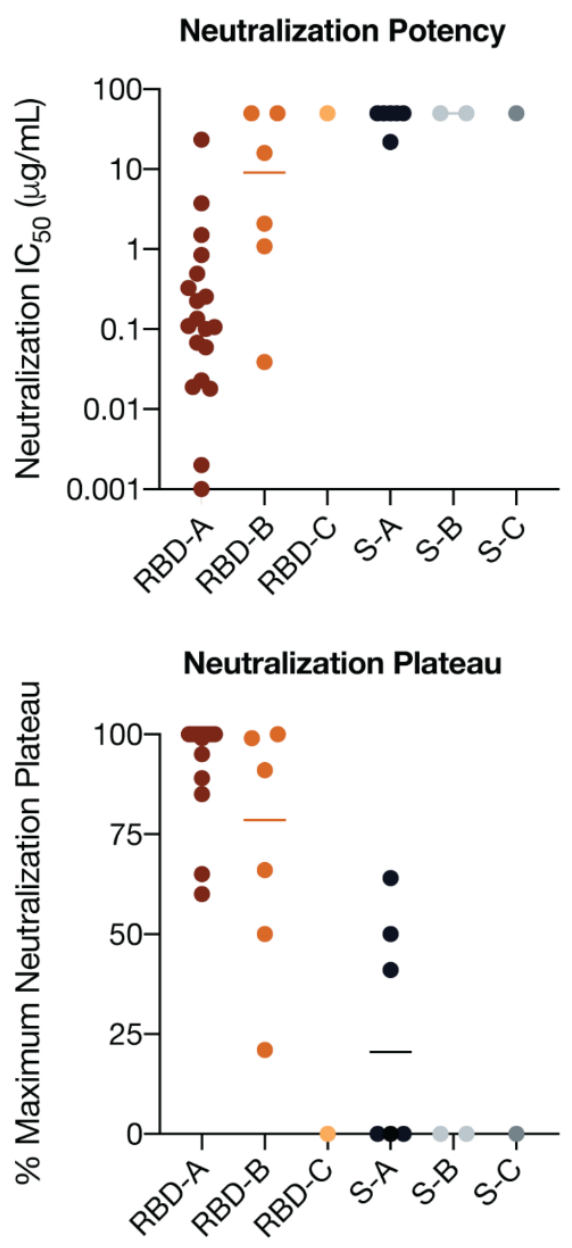

G

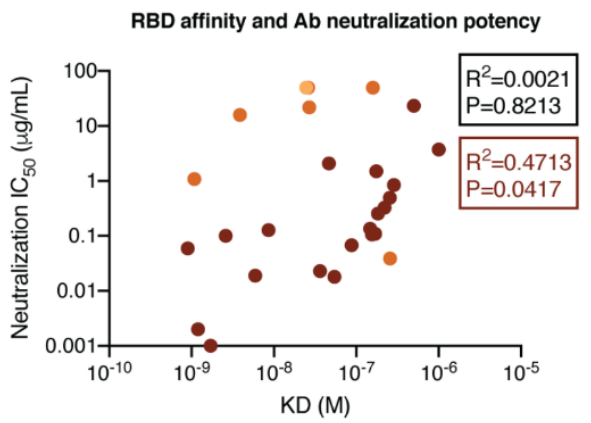

Fig. 4. Antibody functional activity by epitope specificities. Monoclonal antibody epitope binning was completed using RBD and SARS-CoV-2 S protein as target antigens. (A) A total of three non-competing epitopes for RBD (RBD-A, RBD-B, and RBD-C) and three non-competing epitopes for S (S-A, S-B, and S-C) were identified. (B) MAbs were evaluated for binding to different target antigens (S, N-terminal domain (NTD), RBD, RBD-SD1, and RBD-SD1-2) by ELISA and apparent EC50s are reported as $\mu \mathrm{g} / \mathrm{ml}$. (C) MAbs were evaluated for neutralization of SARS-CoV-2 pseudovirus using HeLa-ACE2 target cells. Antibodies are grouped according to epitope specificities and neutralization IC50 values are reported as $\mu \mathrm{g} / \mathrm{ml}$. (D) The maximum plateaus of neutralization (MPN) are reported for each $\mathrm{mAb}$ and grouped by epitope specificity. MAbs were mixed with (E) S or (F) RBD protein and measured for binding to HeLa-ACE2 target cells as a measure of competition to the cell surface ACE-2 receptor. (G) mAb neutralization potencies (IC50, $\mathrm{gg} / \mathrm{ml}$ ) are plotted as a function of dissociation constants (KD, M) measured by surface plasmon resonance (SPR) to RBD target antigen. 
A

i.p i.n
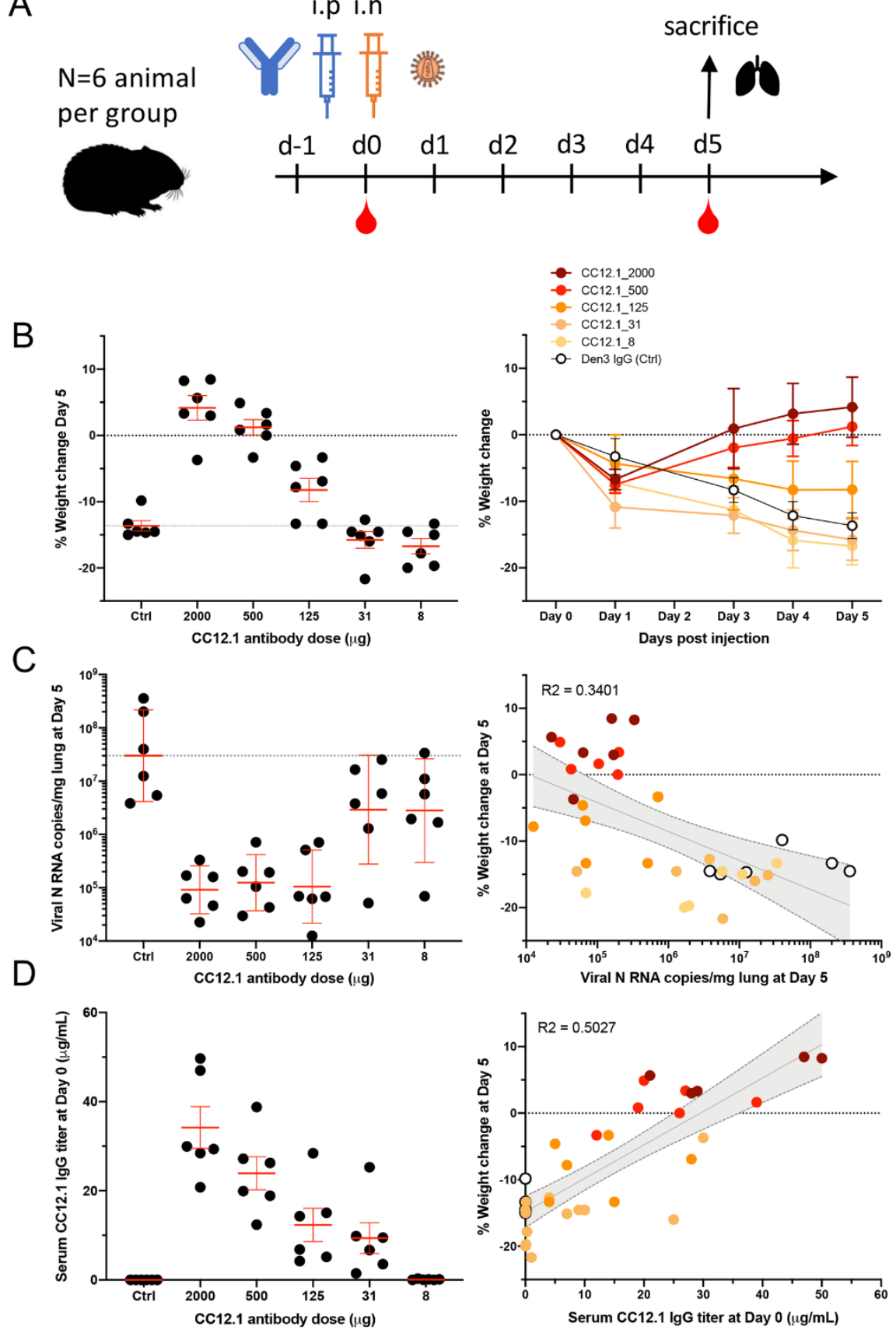

Fig. 5. A potent SARS-CoV-2 RBD-specific neutralizing mAb protects against weight loss and lung viral replication in Syrian hamsters. (A) SARS-CoV-2specific human neutralizing $\mathrm{mAb} C \mathrm{C} 12.1$ isolated from natural infection was administered at a starting dose of $2 \mathrm{mg} /$ animal (on average $16.5 \mathrm{mg} / \mathrm{kg}$ ) and subsequent serial 4 -fold dilutions. Control animals received $2 \mathrm{mg}$ of Den3. Each group of 6 animals were challenged intranasally 12 hours post-infusion with $1 \times 10^{6}$ PFU of SARS-CoV-2. Serum was collected at the time of challenge Day 0, and their weight monitored as an indicator of disease progression. On day 5 , lung tissue was collected for viral burden assessment. (B) Percent weight change was calculated from day 0 for all animals (C) Viral load as assessed by Nucleocapsid RNA q-PCR from lung tissue at day 5 post infection. (D) Serum titers of the passively administered mAb, as assessed by ELISA at the time of challenge (12 hours after i.p administration). Correlation analyses with 95\% confidence intervals indicated in grey shade. $R^{2}$ values are also indicated. 